\title{
Is Bray-Curtis differentiation meaningful in Molecular Ecology?
}

\author{
William Sherwin ${ }^{1}$ \\ ${ }^{1}$ Univ of NSW
}

April 14, 2021

\begin{abstract}
A popular measure of differentiation in biodiversity is the Bray Curtis index of dissimilarity. It has recently also been proposed for use in molecular ecology. However, this measure currently cannot be predicted under specified conditions of population size, dispersal and speciation or mutation. Here I show forecasts for Bray-Curtis for two-variant systems such as single-nucleotide polymorphisms (SNPs) (or two species ecosystems). These are derived from well-known equations in population genetics, for forecasting measures such as G_ST, and shown to be appropriate by simulation. Thus, Bray-Curtis can now be used for assessment of differentiation, in order to understand natural or artificial processes, thus complementing other measures with different sensitivities, such as Morisita-Horn/D_EST, G_ST and Shannon Mutual Information/Shannon Differentiation.
\end{abstract}

\section{Hosted file}

Bray MS 210303refs.pdf available at https://authorea.com/users/407719/articles/517957-isbray-curtis-differentiation-meaningful-in-molecular-ecology 